\title{
REALIZATION OF A SINGLE IMAGE \\ WITH AUTOCOLLIMATION FOR PHA I
}

\author{
Xu Jiayan Wang Hongqi Zhang Zhiwu \\ Shaanxi Astronomical Obs., Academia Sinica \\ P.0. Box 18, Lintong \\ Xian, P.R. China
}

ABSTRACT. The First PHotoelectric Astrolabe ( PHA I ) of China was installed at Shaanxi Obs. in 1971 and came into use later. PHA I has been operating automatically since 1986. Now it is being revised by using a single image with autocollimation, equipped with photon counting system and a tracking diaphragm with a small window to follow the star in zenith direction, which would cut off the most light of the night sky. With all these three approaches, PHA I could improve its limiting magnitude from mag. 6 to mag. 11 or even fainter.

\section{Introduction}

In the early 1950s, well known French astronomer A. Danjon completed great improvements on the prism astrolabe made by A. Claude and L. Driencourt, and then Danjon Astrolabe became precise tool in astrometry (Danjon, 1955) and were used in the determination of both the Earth rotation parameters and star position correction in the world. In order to fit the increasing needs of modern astrometry, the First PHotoelectric Astrolabe (PHAI) with the aperture of $150 \mathrm{~mm}$ was made in China and installed at Shaanxi 0bs. in 1971 (Elaborate Group of Photoelectric Astrolabe, 1973; First Division of Shaanxi 0bs., 1975). As successor of PHA I, three PHI IIs with the aperture of $200 \mathrm{~mm}$ were used at Shanghai 0bs. in 1974, and at Beijing 0bs. and Yunnan 0bs. later (Elaborate Group of Photoelectric Astrolabe, 1975). Also France and Japan made their own photoelectric astrolabe in 1980s (Billaud et al., 1982; Tsubokawa et al.,1984). The PHA II with a special combined prism does provide very stable zenith distance of the almucantar, which benefits the determination of the star declination greatly (First Division of Shanghai Obs., 1976; 1977).

PHA I has been operating automatically since 1986 (Wang Hongqi et al., 1987). and now it is being revised with photon counting detector and with a single image by using autocollimation as well as a diaphragm to track the star in the zenith distance. In this way, it could hopefully observe faint stars up to mag. 11 or even fainter.

\section{Realization}

The principle of a single image for PHA $I$ is shown in Figs.1 and 2. Fig.1 shows all the optical parts. In order to observe stars at two different 
almucantars and to get absolute declination of stars, we would use the front face ( $A$ for zenith distance of $30^{\circ}$ ) and rear face ( $B$ for zenith distance of $45^{\circ}$ ) of the combined prism ( see Fig.1). So our version of the single image with autocollimation for PHA I has the following features:

1) The light source of autocollimation and the slit for detecting the image of autocollimation together with the grid for recording star transit are in the same glass plate located in the focal plane ( see Fig.2), which would make the system very stable;

2) The combined prism could rotate $180^{\circ}$ around a vertical line automatically driven by a stepping motor within a minute, so we could use either face A or B of it at any time;

3) The photon counting technique is used to determine the position of the almucantar with the precision of a few hundredths of $\operatorname{arcsec}(\mathrm{as})$;

4) The variation of the position of the almucantar in the grid is used to drive the plate to its correct position in time and to correct the recording time of the star transit after observations if it is larger than a certain value ( say 0.01 as);

5) The angular position of the plate ( 6 in Fig.1) measured by a circular inductosyn ( 7 in Fig.1) with the resolution of 11 as, which corresponds to the resolution of 0.01 as in zenith distance, is given by a digital encoder;

6) The light of the night sky is reduced to about 300 square as, which is much less than original version with double images, because a diaphragm with a hole of 50 (as) $* 6$ (as) is used to track star image in zenith direction;

7) The aperture for a single image has increased by about 2.4 times over the old version (with consideration of the reflectivity of the mercury being about $75 \%)$.

The special recording grid ( shown in Fig.2) with a pin-hole ( 2 in Fig.2) on one side and a horizontal slit ( 3 in Fig.2) on other side is used to measure the position of the almucantar and the time of the star transit. The autocollimation light from the pin-hole goes backwards to the star light, and after reflection by the small prism (2 in Fig.1) and the mercury ( 3 in Fig.1), it goes back with the star light, and forms the image on other side of the grid. When the plate ( 6 in Fig.1) driven by the stepping motor rotates clockwise and counter-clockwise with the amplitude of $5^{\circ}$, the autocollimation image moves up and down with the amplitude of about 17 as. This can be shown in Fig. 3. When the plate is vertical, the autocollimation image should be in the middle line of the slit under the assumption that the normal of the mirror is parallel to the optical axis of the teiescope. When the plate tilts by an angle of $\theta$ from the vertical,the autocollimation light tilts with respect to optical axis by a small angle $\Delta \alpha$, as if the light source went up by $\Delta h=d * \tan \theta *(n-1) / n$ ( $d$ is the thickness of the plate, $n$ is the refractivity of the glass plate). After reflection by the mirror, the light comes back and forms an image below its source by $\Delta H=\Delta h+\Delta h^{\prime}$, where $\Delta h^{\prime}=d \star \tan \theta^{\prime}(n-1) / n, \theta^{\prime}=\theta-\Delta \alpha$. If adopting $\mathrm{d}=5 \mathrm{~mm}, \mathrm{n}=1.5163$, we could obtain $\Delta \alpha=\Delta \mathrm{h} / \mathrm{F}=8.535$ as, $\Delta \alpha^{\prime}=\Delta \mathrm{h}^{\prime} / \mathrm{F}=8.527$ as, and $\Delta \mathrm{H} / \mathrm{F}=17.06$ as.

When the autocollimation image passes through the slit due to the plate 
rotation, a photomultiplier behind the slit yields some photons which contain the information of the almucantar position. Three times of autocollimation for a star transit would be used: before (A in Fig.2), after (C) and in the middle (B) of observation of the star. One cycle of rotation of the plate will take 0.5 seconds and give 2 peaks of photons. $n$ peaks $(n=6$ or 4$)$ will be used to determine the position of the almucantar in one time of autocollimation. We would sample both the photons of the autocollimation image and the angle of the plate during autocollimation at exact same moment given by the sampling pulses with the cycle of 2 or 1 milliseconds. In order to get the correct position of the almucantar, the method of convolution similar to one used by E. Hog ( 1970 ) will be used to determine the position of the maximal point for each peak of the photon waves with the precision of a fraction of the span over two adjacent samples $\mathrm{i}$ and $\mathrm{i}+1$ ( see Fig.4), then the position of the maximum of each peak will be used to find the angular position, $\theta_{j}$, of the plate with same precision ( see (b) in Fig.4), which corrensponds to the position of the almucantar. Averaging all the $\theta_{i}(j=1,2, \ldots n, n=6$ or 4$)$ gives the final position of the almucantar: $\theta_{0}=\sum \theta_{j} / 6$, which will be used to drive the plate to its correct position in time and to correct the recording time of the star transit later. Another photomultiplier behind the grid is used for detecting the star image, from which the photons will be treated, and it gives the time of the star transit after observations at each night.

For PHA $I$, because the aperture of the main mirror $(150 \mathrm{~mm})$ is divided into two pupils, each of them is equivalent to an aperture of $75 \mathrm{~mm}$. Since the mercury has only reflectivity of $70-75 \%$, so its pupil works as an aperture of $55 \mathrm{~mm}$. The aperture used by the single image is about $135 \mathrm{~mm}$, almost equa! to the diameter of the main mirror. The rest part of the main mirror is used for autocollimation. In this way, we could increase the aperture for star image by 2.4 times over the old version with double images. In addition, we would add a diaphragm driven by a stepping motor in front of the grid, which follows the star up or down in the zenith direction with the precision of a few arcsec. In this way, the most of the light of the night sky will be cut off, and another 1 or 2 mags. improvements will be obtained. After these two measures together with the photon counting technique are adopted, PHA I would hopefully observe faint stars up to mag. 11 or even fainter, and benefit the work of the star catalogue greatly.

\section{Status}

We should have finished this work by the end of last year. Because the manufacture of the combined prism was delayed almost one year, we now try to install all the parts in PHA $I$ by the end of this year or early next year. The simulating calculation shows us that the position of the almucantar could be determined with the precision of 0.01-0.02 as level. 


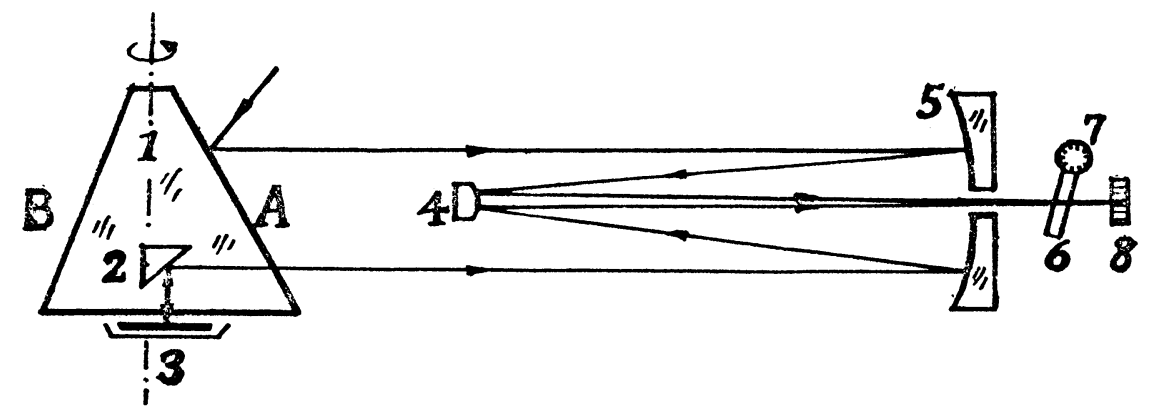

1. Big prism

2. Small prism
3. Mercury plate

4. Secondery mirror

5. Primary mirror

6. Modulation plat
7. Inductosyn

8. Grid plate

Fig. 1 The optical scheme of PHA I for the single image with autocollimation

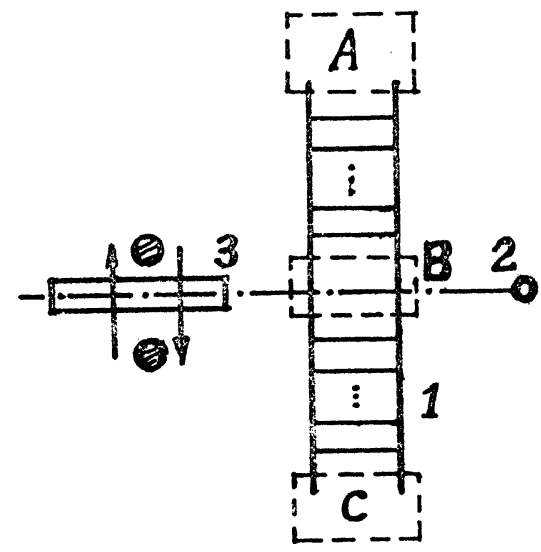

1. Recording grid for stars

2. Pin-hole of the light source

3. Slit for autocollimation image

4. A, B, C: autocollimation time

Fig.2 The grid plate

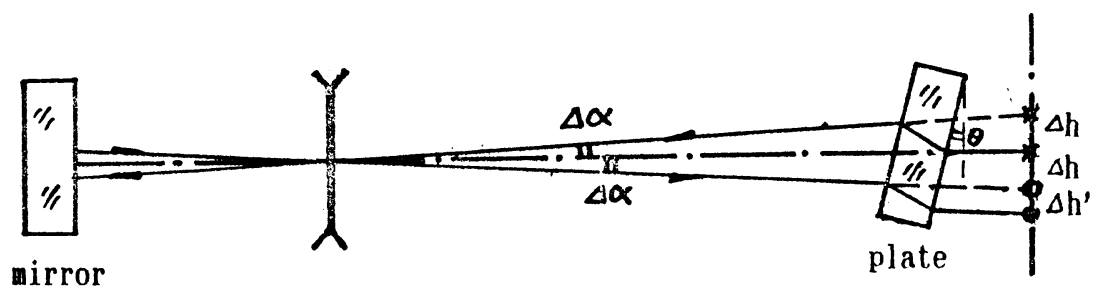

Fig.3 Relationship between the plate tilt and the motion of autocollimation image 
(a)

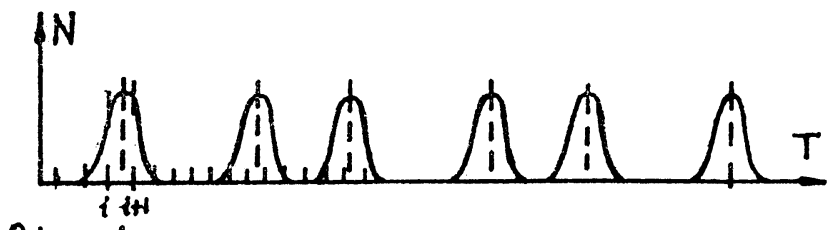

(b)

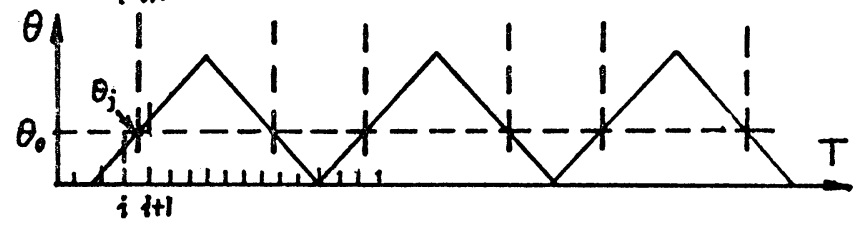

Fig. 4 Waves of the photons sampled of autocollimation image scanning the slit due to the plate rotating and the angular position of the plate sampled at same moment

\section{References}

Danjon, A.: 1955, L'astrolabe impersonnel de l'observatoire de Paris. Bull. Astron. XV.4, 262.

Elaborate Group of Photoelectric Astrolabe: 1973, Chinese Science Bulletin, 5. 216 .

Elaborate Group of Photoelectric Astrolabe: 1975, ACTA ASTRONOMICA SINICA, 16,115 .

First Division of Shaanxi Observatory: 1975, ACTA ASTRONOMICA SINICA, 16, 104.

First Division of Shanghai Observatory: 1976, ACTA ASTRONOMICA SINICA, 17, 27.

First Division of Shanghai Observatory: 1977, ACTA ASTRONOMICA SINICA, 18, 4.

Billaud, G., Texereau, J.: 1982, C. R. Acad. Sc. Paris, t.295, 669.

Tsubokawa, I. et al.: 1984, Publ. Int. Latit. Obs., Mizusawa, 18, 105.

Wang Hongqi, Zhang Zhiwu, Xu Jiayan: 1987, Publication of Shaanxi Observatory, 10,29 .

Hog, E.: 1970, Astron. and Astropys., 4, 89. 